\title{
Editor's Message to Special Issue on Embedded Systems Engineering
}

\author{
YUKIKAZU NAKAMOTO ${ }^{1, a)}$
}

Embedded systems are important components supporting the social life of the people as a wide variety of products, such as automobiles, home appliances, industrial equipments and telecommunication devices and the foundation of industries in Japan. Embedded system research and development are required to integrating a wide range of multi-dimensional technologies from hardware to software, from basic technologies to applications with innovative ideas since embedded systems provide new services and features within physical and economical constraints. Establishing the embedded systems engineering with a wide range of activities and advancing it becomes not only solutions in the industry but an important issue in order to strengthen competitiveness. In addition, overseas, mainly in Europe and the U.S., research activities for the embedded systems have been activated rapidly and a variety of outcomes have been published. It is necessary to vigorously promote research being carried out in universities and research institutions in Japan.

In this special issue, we call for papers on a wide range of areas from industry and academia. This includes a wide range of themes, such as hardware and software technologies related to embedded systems, project managements, and human resources and educations in addition to papers presented "Embedded System Symposium 2011 (ESS 2011)," which is sponsored by Special Interesting Group on Embedded System in Information Processing Society of Japan,

The number of submitted paper is 27 and 11 excellent papers are accepted. Reviewers who were assigned from the editorial committee as shown below conducted peer-reviewing. Acceptance ratio is $41 \%$.

We should accurate to establish embedded system technologies with cooperation between industry and academia. Publishing special issue which summarizes the research results is important in the field we would like to continue publishing the special issue in the future.

We would like to express our sincere appreciation to all the authors for their contribution.

\author{
The Editorial Committee
}

- Editor in-Chief:

Editor in-Chief: Yukikazu Nakamoto (University of Hyogo)

- Deputy Chief Editor:

Deputy Chief Editor: Kouichi Ono (IBM Research - Tokyo)

\section{- Editorial Committee:}

Toshiaki Aoki (Japan Advanced Institute of Science and Technology)

Masato Edahiro (Nagoya University)

Hiroaki Fukuda (Shibaura Institute of Technology)

Katsuhiko Gondow (Tokyo Institute of Technology)

Mikio Hashimoto (Toshiba Corp.)

Eiichi Hayakawa (Takushoku University)

Tomoji Kishi (Waseda University)

Keiji Kimura (Waseda University)

Hironori Nakajo (Tokyo University of Agriculture and Technology)

Hiroki Matsutani (Keio University)

Nozomu Togawa (Waseda University)

Takanori Yokoyama (Tokyo City University)

Hironori Washizaki (Waseda University)
1 University of Hyogo, Kobe, Hyogo 650-0044, Japan
a) nakamoto@ai.u-hyogo.ac.jp 